\title{
Study of Neutron-Induced Ionization in Helium and Argon Chamber Gases
}

\author{
D. Indurthy ${ }^{a}$ A.R. Erwin ${ }^{c}$ D.A. Harris ${ }^{b}$ S.E. Kopp ${ }^{a, *}$ \\ M. Proga ${ }^{a}$ R.M. Zwaska ${ }^{a}$ \\ ${ }^{a}$ Dept. of Physics, University of Texas, Austin, Texas 78712 U.S.A. \\ ${ }^{\mathrm{b}}$ Fermi National Accelerator Laboratory, Batavia, Illinois 60510 U.S.A. \\ ${ }^{\mathrm{c}}$ Dept. of Physics, University of Wisconsin, Madison, Wisconsin 53706 U.S.A.
}

\begin{abstract}
We present studies of Helium- and Argon-filled ionization chambers exposed to intense neutron fluxes from $\mathrm{PuBe}$ neutron sources $\left(E_{n}=1-10 \mathrm{MeV}\right)$. The sources emit about $10^{8}$ neutrons per second. The number of ion pairs in the chamber gas volume per incident neutron is derived. While limited in precision because of a large gamma ray background from the PuBe sources, our results are consistent with the expectation that the neutrons interact purely elastically in the chamber gas.
\end{abstract}

Key words: ionization chambers, neutron scattering, elastic scattering, electrical phenomena in gases

PACS: 25.40, 29.40, 51.50

* Corresponding author e-mail kopp@mail.hep.utexas.edu 


\section{Introduction}

The Neutrinos at the Main Injector (NuMI) beamline at the Fermi National Accelerator Laboratory [1] will generate an intense $\nu_{\mu}$ beam from the decays of mesons produced in the collisions of $120 \mathrm{GeV}$ protons on a graphite target. The mesons are focused into a $675 \mathrm{~m}$ long evacuated volume where they decay to neutrinos. The meson decays $\pi / K \rightarrow \mu \nu_{\mu}$ produce an energetic muon for every neutrino, allowing monitoring and validation of the neutrino beam focusing to be accomplished by monitoring of the muon flux. As in several previous experiments, the muon flux and remnant hadron flux at the end of the decay volume will be measured by arrays of ionization chambers $[2,3,4,5]$.

In addition to charged particle fluxes, the ionization chambers are exposed to large fluences of neutrons, primarily produced in the aluminum/steel beam absorber upstream of the first muon monitor (see Figure 1). The neutron fluxes, as estimated by a GEANT[6] simulation of the beamline, are as great as $13 \times 10^{9} / \mathrm{cm}^{2} /$ spill at the hadron monitor and $9 \times 10^{7} / \mathrm{cm}^{2} /$ spill in the first muon station. The neutron fluences are a factor of 10 larger than the charged particle levels the chambers should measure. Most of the neutrons are low energy, with a mean energy of $30 \mathrm{MeV}$. The response of the ion chambers to a charged particle flux has been studied with electron and proton beams $[7,8]$. In this article, we study the response of ion chambers to neutrons.

Previous neutrino beamlines have also utilized ionization chamber arrays to measure the muon beam profile and rate. Because of background particles produced in the beam dump, previous experiments have reported having to contend with higher-than-anticipated fluxes $[9,10]$, or even to disregard the rate information from the muon detectors altogether [11].

Unlike charged particles, neutrons do not ionize the gas directly. Instead, fast neutrons interact with nuclei and create a charged recoil nucleus. This nucleus very quickly deposits its energy through ionization. The recoil particle can be produced either in the chamber gas or in the plates of the chamber electrodes.

We studied the response of four parallel plate ion chambers to neutron fluxes at the University of Texas Nuclear Engineering Teaching Laboratory. The neutrons for this test are provided by $\mathrm{PuBe}$ radionuclide sources, which produce 1-10 MeV neutrons via the capture on Be of alpha particles emitted by the $\mathrm{Pu}[12]$. The total source activity is approximately $55 \mathrm{Ci}$, leading to approximately $1.5 \times 10^{8}$ neutrons/sec. The objective of this test is to determine the ionization due to neutron flux, an effective measurement of the cross section for neutron scattering times the ionization left by the recoiling particles. 


\section{Experimental Setup}

The four parallel plate ion chambers are housed in two separate Aluminum gas vessels (see Figure 2). One vessel contains two chambers with $2 \mathrm{~mm}$ gas electrode separation, the other has two chambers with $5 \mathrm{~mm}$ electrode separation. Electrical feedthroughs to the ion chambers are made using PEEK insulating plastic and Aluminum compression fittings. A grounded Aluminum mesh inside the vessel separates the two chambers. The parallel plate ion chambers are fabricated from $1 \mathrm{~mm}$ thick $100 \times 100 \mathrm{~mm}^{2}$ ceramic plates with electrodes made of Pt-Ag alloy, $10 \mu \mathrm{m}$ in thickness. The sense plane has a central $80 \times 80 \mathrm{~mm}^{2}$ collection electrode with a $10 \mathrm{~mm}$ wide guard ring. The chamber materials are identical to that to be used in the NuMI beam monitoring chambers.

One ion chamber in each vessel has a $1 \mu \mathrm{Ci} \mathrm{Am}^{241} \alpha$ source mounted such that the $\alpha$ particles enter its gas volume. The alpha sources permit calibration of temporal effects such as barometric pressure variations. Each vessel also has a signal feedthrough with no internal connection to an ion chamber. These "dummy" feedthroughs serve to check for spurious signals.

Signals and high voltage are delivered to the setup through a shielding wall via coaxial cable with kapton insulator. The exterior of all feedthroughs is potted with epoxy, then wrapped in Aluminum foil to provide a complete grounded shield. This exterior shielding reduces stray electric fields from the high voltage feedthroughs to the signal feedthroughs which can drift ionized charges in the surrounding air to the signal lines.

The chambers are tested under Helium or Argon gas flow at 1000 mbar. The gas used in this test is rated as $99.998 \%$ pure and is passed through an Oxygen getter ${ }^{1}$ before going to the chambers which are connected in series to the gas delivery. The exhaust gas is monitored with an Oxygen analyzer ${ }^{2}$, showing 0.5-1.5 ppm of $\mathrm{O}_{2}$ impurity level during the tests.

The ionization current of each chamber is read by a picoammeter. ${ }^{3}$ The nominal sensitivity of the digital display of a picoammeter is $1 \mathrm{pA}$, however we found that by using the voltage output of the current-to-voltage amplifier we could achieve a sensitivity of better than $0.1 \mathrm{pA}$ for steady currents. The reason for the $1 \mathrm{pA}$ rating of the picoammeter appears to be drift in the internal amplifier circuit which occurred sporadically during the tests. With frequent monitoring of the signals, we could discard data runs in which non-physical drifts are observed. The picoammeter output is digitized by an ADC (least significant bit is equal to $0.06 \mathrm{pA}$ ) and read into a computer.

\footnotetext{
1 Matheson model MTRP-0012-XX

2 Illinois Instruments, model 2550

3 Keithley model 480
} 


\section{Neutron Source}

The neutrons for this test are obtained from six individual $\mathrm{PuBe}$ sources. Four of the sources are ${ }^{239} \mathrm{Pu}$ totalling 8.99 Curies. The two others provide $46 \mathrm{Ci}$ of ${ }^{238} \mathrm{Pu}$. The neutron yield from the ${ }^{239} \mathrm{Pu}$ is measured to be 57 per $10^{6}$ alpha decays, but calculations suggest 65 per $10^{6} \alpha$ decays [13]. Given that yields may vary for specific alloy samples [12], we take $(60 \pm 5) \times 10^{-6}$ neutrons $/ \alpha$. The theoretical neutron yield for ${ }^{238} \mathrm{Pu}$ is $(79 \pm 5) \times 10^{-6} n / \alpha$ [14]. We estimate all sources combined to emit $(1.57 \pm 0.13) \times 10^{8}$ neutrons $/ \mathrm{sec}$.

During the test the neutron sources are moved to several locations around the gas vessels. The neutron flux through each chamber in these runs is estimated using the source activities and the solid angle subtended by the chambers. In performing the solid angle integral over the ion chamber faces, each PuBe source is assumed to be point-like. The neutron flux is quoted in units of pA, where $1 \mathrm{pA}=6.2 \times 10^{6}$ neutrons $/ \mathrm{sec}$. The fluxes through each chamber for each run are tabulated in Table 1.

In Table 1 is calculated as well a cosine-weighted flux. A neutron traversing the chamber at normal incidence receives a weight 1.0, while a neutron entering

at $45^{\circ}$ to the normal receives an extra weight of $\sqrt{2}$. This cosine factor weights for the pathlength of the neutron passing through the ion chamber gas gap at non-normal incidence, which increases its probability of interaction. The error in the fluxes due to the estimation method is about $10 \%$ for the smaller fluxes and $20 \%$ for the largest, which are very sensitive to the source positions.

The $\mathrm{PuBe}$ source also emits gamma rays, which form a background for the measurements of this paper. In approximately $57 \%$ of ${ }^{9} \mathrm{Be}(\alpha, n)^{13} \mathrm{C}$ captures which lead to an emitted neutron from the $\mathrm{PuBe}$ source, the ${ }^{13} \mathrm{C}^{*}$ excited state emits a $4.4 \mathrm{MeV}$ gamma [15]. These $\gamma$ 's may Compton scatter in our chamber materials, yielding additional ionization from the Compton electron. This background is discussed further in Section 5 .

\section{Plateau Measurements}

In each of the runs, the bias voltage for each chamber is ramped from zero volts until gas amplification is observed. The plateau ionization currents from Runs 0 and 10, in which no neutron sources are present, are subtracted from the plateau ionization currents observed in subsequent runs to determine the neutron-induced ionization in the gas. As expected, only the chambers with an alpha source show an appreciable signal during Runs 0 and 10. All chambers show an additional signal when significant neutron fluxes are present. 
Because of voltage offsets in the the current-to-voltage amplifier in the picoammeters, the definition of 'zero ionization current' is determined by measuring the chamber plateau curve with both positive and negative bias voltages and assuming the plateau values to be symmetrical about zero signal. This definition also calibrates away the effect of any contact potentials in the chambers, which cause ionization current to be collected even with zero applied bias voltage. Figure 3, for example, shows the voltage plateau curves for the four chambers during different runs taken with Argon gas flow. While the $2 \mathrm{~mm}$ chambers are fairly symmetric about zero ionization current, the $5 \mathrm{~mm}$ chambers show either some effect of a contact potential or an offset in the picoammeters.

Figures 3 and 4 show the plateau curves for the runs taken with Argon and Helium gas flow, respectively. In the Helium data, the negative chamber bias data is not always available. Therefore, only the positive voltage portion of the He runs is plotted, where the voltage plateaus have been scaled by the ratio of positive and negative ionization currents observed in other runs. The inavailability of the positive and negative polarities in some Helium runs leads to somewhat larger uncertainties in the ionization currents on plateau.

To deduce the ionization current due to the PuBe source, we subtract from the ionization current of a given run the ionization current observed in Run 0 (He data) or Run 10 (Ar data). The chambers without alpha sources are therefore more sensitive to small $\mathrm{PuBe}$ signals. The ionization current attributed to $\mathrm{PuBe}$-induced interactions is plotted as a function of neutron flux in Figures 5 and 6 for the Argon and Helium data, respectively.

We have fit the data in Figures 5 and 6 to find the average PuBe sourceinduced ionization per neutron. The fits constrained to go through the origin gives slope values indicated in Table 2. Allowing the vertical intercept to float in the fit changes the slope in the Helium fits, for example, by only 0.03 ionizations/neutron, and the fitted vertical intercepts are consistent with zero (0.04 \pm 0.05 and $0.05 \pm 0.05$ ionizations for the $5 \mathrm{~mm}$ and $2 \mathrm{~mm}$ chambers, respectively).

\section{$5 \quad$ Expected Rates}

As stated above, some of the observed ionization per neutron in Figures 5 and 6 and in Table 2 is due to the accompanying $4.4 \mathrm{MeV} \gamma$ 's from the PuBe source. In this section we estimate the ionization rate induced by the $\mathrm{PuBe}$ source, accounting separately for (1) the ionization from recoil gas molecules scattered elastically by the PuBe neutrons; and (2) the ionization from Compton electrons ejected by the $4.4 \mathrm{MeV} \gamma$ 's. "Wall effects," whereby $\gamma$ rays are 
produced by inelastic interactions of neutrons in the chamber wall materials [17], are not considered. Similar estimates of neutron scattering in gaseous chambers have been used to estimate rates in drift chambers downstream of a beam dump [19]. The results of the calculations are summarized in Table 4.

\subsection{Neutron Rate}

The estimate of the ionization expected from neutron recoils in the gas first requires the measured total cross section for neutron scattering on Helium or Argon in the energy range $2-40 \mathrm{MeV}$ [18], which are repeated in Table 3 . These cross sections indicate an interaction length $\lambda=(1 / n \sigma)$ of $1-2 \times 10^{4} \mathrm{~cm}$ in the chamber gas. The scattering is predominantly elastic below $10 \mathrm{MeV}[12]$.

The mean energy of an elastically-scattered nucleus is $\bar{E}_{R}=\left(2 A /(1+A)^{2}\right) E_{n}$, where $E_{R}$ is the recoil nucleus energy, $E_{n}$ the incident neutron energy, and $A$ the target nucleus mass [12]. These recoil energies, along with the estimated ranges of these recoils in the chamber materials, are listed in Table 3. The ranges are extrapolated from measured ranges of alpha particles [20] using the

scaling relation [21] $R=R_{\alpha}\left(m / m_{\alpha}\right)\left(\sqrt{z_{\alpha} / z}\right)$, where $m$ and $z$ are the mass and charge of the recoiling nucleus, $R_{\alpha}$ is the range of an alpha at the same velocity as the nuclear recoil.

The ranges of recoil $\mathrm{Al}, \mathrm{O}, \mathrm{Pt}$, or $\mathrm{Ag}$ nuclei from the walls are insufficient to traverse the chamber wall materials and reach the chamber gas volume, with the exception of Pt ions liberated very near the electrode surface. Even the Pt ions will contribute insignificant ionization in the chamber gas, since the $\mathrm{Pt}$ ions' energies are quite low. In fact, the utility of Pt electrodes for reducing "wall-effects" in neutron interactions has been noted previously [22].

The ionization of the recoils created in the gas is then calculated. Because of the short $(1.2 \mathrm{~mm})$ range of recoil Ar ions in Ar gas, we estimate their ionization as the recoil energy divided by the energy $w=27 \mathrm{eV}$ to create an ionization in Ar gas [23], yielding an average of 8900 ionizations. For Helium recoils, we take $d E / d x=380 \mathrm{keV} / \mathrm{cm}[20]$ for $\bar{E}_{R}=1.6 \mathrm{MeV}$ alpha's, divided by $w=32 \mathrm{eV}$ to create an ion pair [24], yielding 11,900 ionizations for a chamber with $1 \mathrm{~cm}$ electrode spacing.

To estimate the average ionization per neutron, we multiply the probability for neutron scattering in the gas by the ionization deposited per recoil. The results are given in Table 4. 


\subsection{Gamma Rate}

To estimate the ionization caused by $\gamma$ rays emanating from the PuBe source, we performed a parametric Monte Carlo calculation of $\gamma$ 's Compton scattering in the chamber wall materials, followed by energy loss and multiple scattering by the Compton-scattered electrons. The ionization induced in the chamber gas volume is calculated for those electrons which arrive there.

Compton scattering of the $4.4 \mathrm{MeV} \gamma$ 's occurs with approximately 1 barn cross section [16]. This yields a probability of scattering in the Aluminum chamber walls of $25 \%$ and a probability of scattering in the ceramic plates of $11 \%$. Including the fact that only $57 \%$ of neutrons are accompanied by a 4.4 $\mathrm{MeV} \gamma$, this yields $19 \%$ of neutron events producing a Compton scatter in the chamber walls.

After the Compton scatter, the electron energy is calculated. Its energy loss is calculated using tabulated stopping power $d E / d x$ data [25]. Multiple scattering of the electron in the Aluminum wall and in the ceramic is estimated as in [16]. If the path through each material (aluminum or ceramic) is greater than the electron's range, the electron does not reach the chamber gas volume. Approximately $74 \%$ of the Compton electrons are either ranged out in the chamber walls or are multiple scattered away from the gas volume.

The $d E / d x$ of the electrons arriving in the chamber gas volume is multiplied by the path length of the electrons through the gas volume. The electron pathlength through the gas can be larger than the electrode separation due to their large multiple scattering. The range of the electrons is $10^{3}-10^{4} \mathrm{~cm}$ in $\mathrm{He}[25]$, so no electrons range out in the gas. For the He gas, we find a mean of 3.7 (8.8) ion pairs created in the $2 \mathrm{~mm}(5 \mathrm{~mm})$ chamber. The results after multiplying by the probability $0.18 \times 0.26$ to have a Compton electron, are shown in Table 4.

\section{Conclusions}

We have studied the signals induced in ionization chambers exposed to low energy (1-10 MeV) neutrons in the form of a PuBe source. An ionization current from the source is observed whose magnitude is $(1.1 \pm 0.2 \pm 0.1)$ ionizations/neutron/cm in the Helium gas, and (9.6 $\pm 2.4 \pm 1.0)$ ionizations/neutron/cm in the Argon gas, based on our observations at several neutron fluences and two chamber gas gaps. The first uncertainty is that due to the estimate of the neutron fluxes, while the last uncertainty is due to the $\mathrm{PuBe}$ source activity into neutrons. 
Our measurements of the PuBe source-induced ionization are limited in precision because of the large number of $\gamma$ rays from the PuBe sources. However, the neutron-specific signal inferred is consistent with that expected from pure elastic scattering of the neutrons in the chamber gas: 0.35 ion pairs per neutron per centimeter in He, and 0.75 ion pairs per neutron per centimeter in Argon. Because of the problem of backgrounds from the $4.4 \mathrm{MeV} \gamma$ 's from the PuBe source, a study of the individual pulse heights for each particle interaction in our chambers is desirable; such will be the subject of further study.

Our measurements indicate the NuMI beam monitors will have a background signal of order $20-30 \%$ from neutron interactions in the chambers. While the yield of ion pairs per neutron is only 2-3\% for that of charged particles (assuming elastic scattering), GEANT[6]-based Monte Carlo estimates indicate that the hadron monitor and the first muon monitor will be exposed to the order of 10 times as many neutrons as signal particles (protons in the case of the Hadron monitor and muons in the case of the muon monitors). The neutron background signal will be much less correllated spatially with beam misalignments than the signal, so its effect will be important to model.

\section{Acknowledgements}

We thank the staff of the University of Texas Nuclear Engineering Department for providing the neutron source facility, and especially Sean O'Kelly and Bill Kitchen, for their valuable assistance. We thank Evan Fuller and James Hall for assistance with the ionization chamber readout. This work was supported by the U.S. Department of Energy, DE-AC02-76CH3000 DE-FG03-93ER40757, and DE-FG02-05ER40896, and the Fondren Family Foundation.

\section{References}

[1] J. Hylen et al. "Conceptual Design for the Technical Components of the Neutrino Beam for the Main Injector (NuMI)," Fermilab-TM-2018, Sept., 1997.

[2] H. Wachsmuth, "Neutrino Beam Monitoring," Proceedings of the Symposium on Beam Intensity Measurement, Daresbury, England, DNPL/RI (1968), pp.6874; R. Bertolotto et al., Proc. 6th Intl. Conf. High Energy Accel., C.E.A., 11-15 Sept. 1967 (CEAL-2000-1967).

[3] R. Blair et al., Nucl. Instr. Meth. A226, 281 (1984).

[4] A. A. Belkov et al., "Calibration of Muon Detectors for IHEP Neutrino Beam Monitoring System," Serpukhov IHEP preprint IFVE-82-99, Apr. 1982, 
Proceedings of the Eigth International Symposium on Nuclear Electronics, Dubna, 1975, pp 392-395.

[5] J. E. Hill et al., "Tests of Beam Monitoring Equipment for a Neutrino Experiment," Beam Sci. Technol. 3 18-22 (1998); Int. J. Mod. Phys. A16S1B, 758-760 (2001).

[6] GEANT Detector Description and Simulation Tool, CERN Program Library, W5013 (1994).

[7] R. Zwaska et al., IEEE Trans. Nucl. Sci. 50, 1129 (2003)

[8] J. McDonald et al., Nucl. Instr. Meth. A496 293 (2003).

[9] N.V. Baggett et al, Nucl. Instr. Meth. 185, 87 (1981).

[10] T. Nakaya, private communication.

[11] P. Auchincloss, A Measurement of the Total Cross Section for Neutrino Nucleon Interactions, $\mathrm{PhD}$ thesis, Columbia University, 1987.

[12] G. F. Knoll, Radiation Detection and Measurement, (New York: John Wiley \& Sons, Inc.), 2000.

[13] K. W. Geiger and L. Van der Zwan, Nucl. Instr. Meth., 131, 315 (1975).

[14] M. E. Anderson and M. R. Hertz, Nucl. Sci. Eng., 44, 437 (1971).

[15] S. Croft, Nucl. Instr. Meth., A281, 103 (1989).

[16] K. Hagiwara et al, Phys. Rev. D66, 010001 (2002)

[17] H. Schraube and H. Bichsel, Radiation Protection Dosimetry 13, 373 (1985).

[18] V. McLane, C. Dunford, and P.F. Rose, eds., Neutron Cross Sections, Vol.2: Neutron Cross Section Curves, National Nuclear Data Center, (Boston: Academic Press, Inc.) 1988.

[19] J. Belz et al., Nucl. Instr. Meth. A428, 239 (1999); S. D. Worm, PhD Thesis, University of Texas, Austin (1995).

[20] ICRU Report 49, "Stopping Powers and Ranges for Protons and Alpha Particles," International Commission on Radiation Units and Measurements, 1993.

[21] R. D. Evans, The Atomic Nucleus, (New York: McGraw-Hill) 1955.

[22] J. H. Coon and R. A. Nobles, Rev. Sci. Instr. 18, 44 (1947).

[23] ICRU Report 31 "Average Energy Required to Produce An Ion Pair," International Commission on Radiation Units and Measurements, 1 May, 1979.

[24] I. Christophorou, Atomic and Radiation Physics, (New York: John Wiley \& Sons) 1973.

[25] ICRU Report 37, "Stopping Powers and Ranges for Electrons and Positrons," International Commission on Radiation Units and Measurements, 1989. 


\begin{tabular}{|c|c|c|c|c|c|c|}
\hline & PuBe & & \multicolumn{4}{|l|}{} \\
Run & Activity & Chamber & \multicolumn{4}{|r|}{ Neutron Fluxes (pA) at Chambers } \\
No. & $(\mathrm{Ci})$ & Gas & $2 \mathrm{~mm} \alpha$ & $2 \mathrm{~mm}$ no- $\alpha$ & $5 \mathrm{~mm} \alpha$ & $5 \mathrm{~mm}$ no- $\alpha$ \\
\hline 0,10 & 0 & $\mathrm{He}, \mathrm{Ar}$ & 0 & 0 & 0 & 0 \\
1,11 & 46 & $\mathrm{He}, \mathrm{Ar}$ & $0.10(0.21)$ & $0.83(0.92)$ & $2.23(2.84)$ & $0.15(0.36)$ \\
2 & 55 & $\mathrm{He}$ & $0.28(0.41)$ & $0.94(1.04)$ & $2.53(3.04)$ & $0.17(0.41)$ \\
3 & 55 & $\mathrm{He}$ & - & - & $3.12(4.00)$ & - \\
4 & 55 & $\mathrm{He}$ & - & - & $3.12(4.00)$ & - \\
$5 \mathrm{a}, 12$ & 46 & $\mathrm{He}, \mathrm{Ar}$ & $0.28(0.66)$ & $1.26(1.46)$ & $1.26(1.46)$ & $0.28(0.66)$ \\
$5 \mathrm{~b}, 13$ & 46 & $\mathrm{He}, \mathrm{Ar}$ & $1.26(1.46)$ & $0.28(0.66)$ & $0.28(0.66)$ & $1.26(1.46)$ \\
6,12 & 46 & $\mathrm{He}$ & $2.23(2.84)$ & $0.15(0.36)$ & $0.10(0.21)$ & $0.83(0.92)$ \\
7 & 55 & $\mathrm{He}$ & $2.53(3.04)$ & $0.17(0.41)$ & $0.28(0.41)$ & $0.94(1.04)$ \\
8 & 55 & $\mathrm{He}$ & $3.12(4.00)$ & - & - & - \\
9 & 55 & $\mathrm{He}$ & $3.12(4.00)$ & - & - & - \\
\hline
\end{tabular}

Table 1

Runs taken during the study of neutron interactions. The gas in the chambers is noted, as well as the expected neutron flux on each of the chambers during each run. The neutron fluxes are quoted in units of 'picoamperes', where $1 \mathrm{pA}=6.2 \times 10^{6}$ neutrons/sec. Both the unweighted and cosine-weighted fluxes (in parentheses) are listed. 


\begin{tabular}{|c|c|c|}
\hline Chamber & \multicolumn{2}{|c|}{ Observed Ion Pairs per Neutron } \\
Gap & He Gas & Ar Gas \\
\hline \hline $2 \mathrm{~mm}$ & $0.26 \pm 0.07$ & $2.4 \pm 0.3$ \\
$5 \mathrm{~mm}$ & $0.49 \pm 0.06$ & $3.7 \pm 0.4$ \\
\hline $1 \mathrm{~cm}^{a}$ & $1.08 \pm 0.15$ & $9.6 \pm 2.4$ \\
\hline
\end{tabular}

Table 2

${ }^{a}$ Inferred from 2, $5 \mathrm{~mm}$ gap chambers.

Ionization current per neutron obtained from fits of the data in Figures 5 and 6. The fitted slopes are equivalent to ion pairs per neutron.

\begin{tabular}{|c|c|c|c|c|c|c|c|}
\hline & & & $\sigma_{\text {tot }}(\mathrm{b})[18]$ & & \multicolumn{3}{|c|}{ Particle Range in Media } \\
Element & $A$ & $Z$ & $E_{n}=2,5,10 \mathrm{MeV}$ & $\overline{E_{R}} / E_{n}$ & $\mathrm{He}(\mathrm{mm})$ & $\mathrm{Ar}(\mathrm{mm})$ & $\mathrm{Pt}(\mu \mathrm{m})$ \\
\hline \hline $\mathrm{He}$ & 4 & 2 & $4.1,2.3,1.5$ & 0.320 & 47 & - & - \\
$\mathrm{Al}$ & 27 & 12 & $3.2,2.3,1.7$ & 0.069 & 12.3 & 1.9 & 0.3 \\
$\mathrm{Ar}$ & 40 & 18 & $4.3,3.4,2.2$ & 0.048 & - & 1.2 & - \\
$\mathrm{Ag}$ & 108 & 47 & $6.5,4.1,4.4$ & 0.018 & 2.6 & 0.27 & 0.1 \\
$\mathrm{Pt}$ & 196 & 78 & $5.8,6.6,5.2$ & 0.010 & 0.74 & 0.13 & 0.1 \\
\hline
\end{tabular}

Table 3

Elastic scattering data for materials found in our ionization chambers, The total cross sections are given for the range $2-10 \mathrm{MeV}$. The average recoil energy is provided as a portion of the neutron's energy. Range is calculated for the typical fully-ionized recoil nucleus of a $5 \mathrm{MeV}$ neutron in both gaseous Helium and Argon and in Platinum using data from [20] and scaling laws from [21].

\section{Table 4}

\begin{tabular}{|c|c|c|c|c|c|c|}
\hline $\begin{array}{c}\text { Chamber } \\
\text { Gap }\end{array}$ & \multicolumn{4}{|c|}{ Expected Ion Pairs per Neutron } \\
\hline & $n$ 's & $\gamma^{\prime}$ s & Total & $n$ 's & $\gamma^{\prime}$ s & Total \\
\hline \hline $2 \mathrm{~mm}$ & 0.07 & 0.18 & 0.25 & 0.15 & 1.8 & 2.0 \\
$5 \mathrm{~mm}$ & 0.18 & 0.44 & 0.62 & 0.37 & 4.4 & 4.8 \\
$1 \mathrm{~cm}$ & 0.35 & 0.84 & 1.19 & 0.75 & 8.4 & 9.2 \\
\hline
\end{tabular}

Expected ionization current per neutron decay from the PuBe source, as described in Section 5. The estimates include the ion pairs produced by elastic scatters of neutrons in the chamber gas ( $n$ 's), as well as those arising from Compton scatters of subsequent cascade $\gamma$ 's from the PuBe source ( $\gamma$ 's). The total expected signals may be compared to the observed values listed in Table 2 . 


\section{Fire Door}

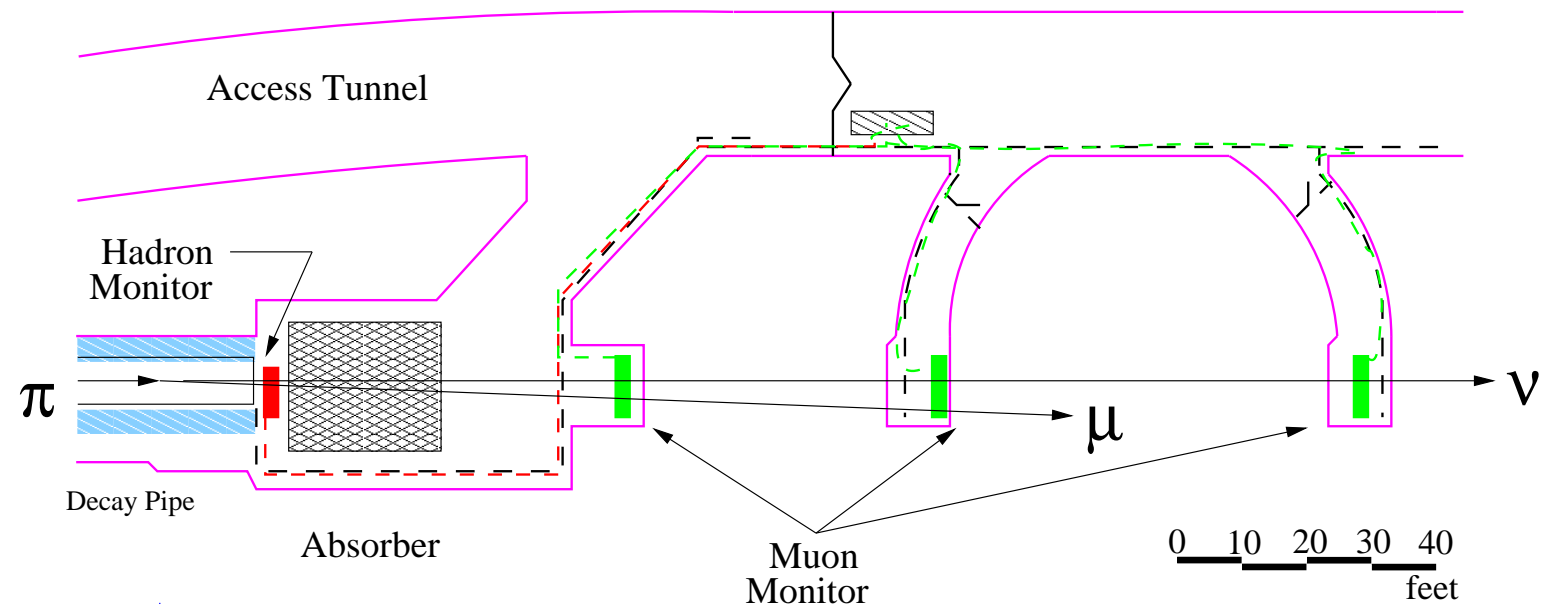

Fig. 1. Location of the four ionization chamber arrays at the downstream end of the NuMI neutrino beamline. Mesons decaying in the evacuated drift volume decay into muons and neutrinos. Ion chamber arrays are located at four stations: upstream of the beam absorber and embedded in the earth in three "alcoves." These arrays monitor the hadron and the muon components, respectively, of the beam. 


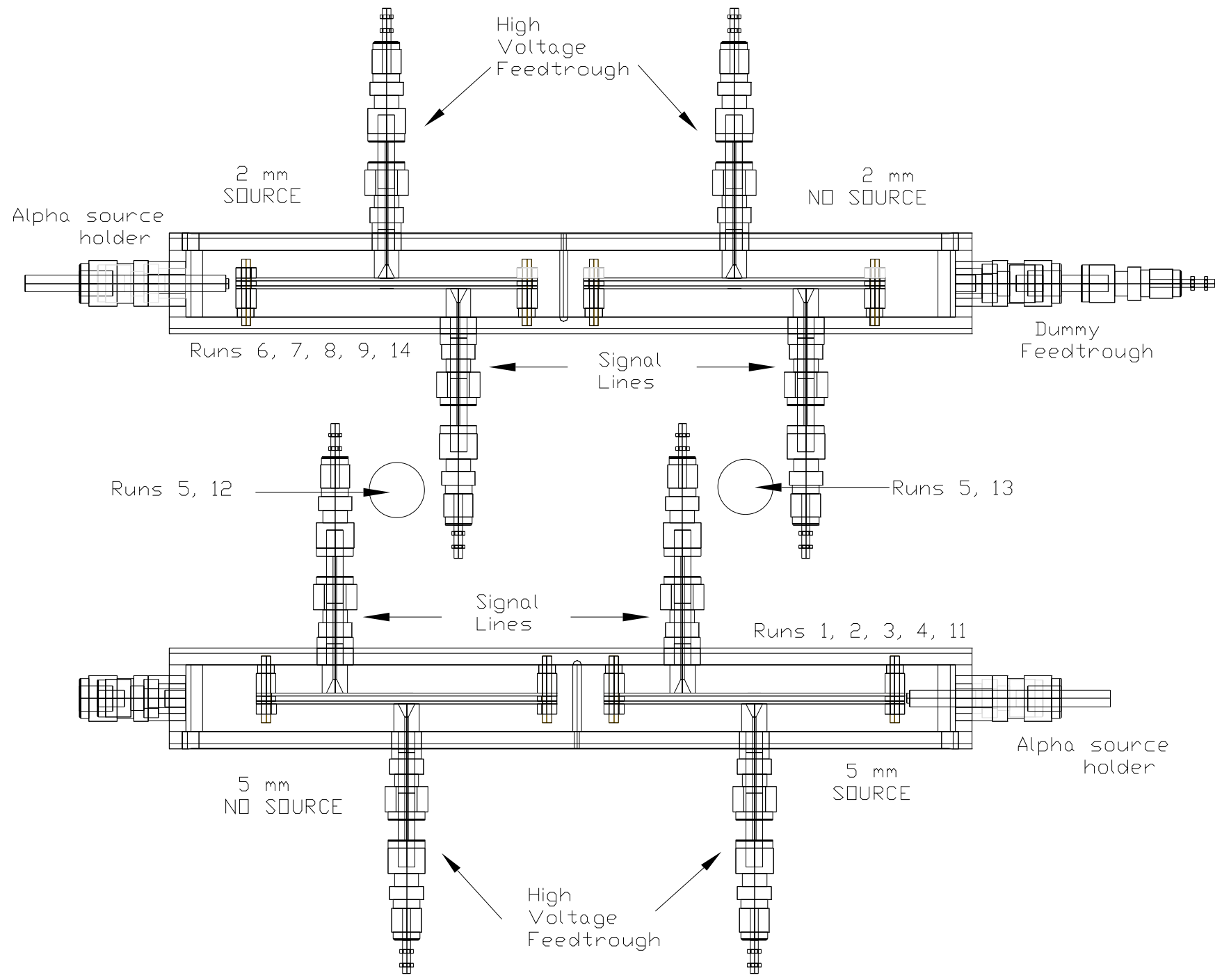

Fig. 2. The apparatus used in this test. Two gas vessels, each with two parallel plate ionization chambers, are placed approximately $20 \mathrm{~cm}$ apart. One vessel has $2 \mathrm{~mm}$ gap chambers and one has $5 \mathrm{~mm}$ gap chambers. The placements of the PuBe sources near the gas vessels for the runs in Table 1 are indicated. 

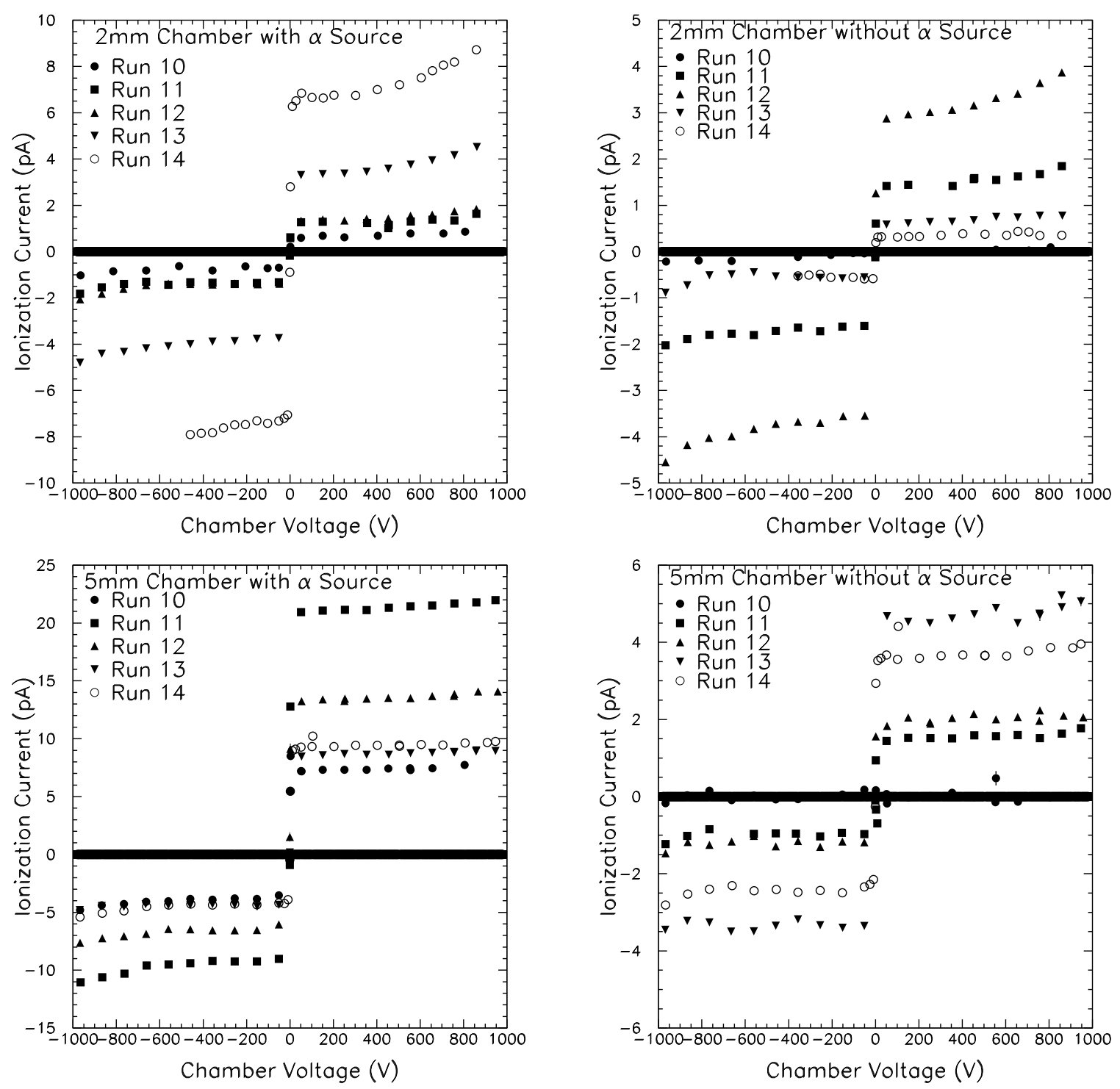

Fig. 3. Plateau curves for $2 \mathrm{~mm}$ and $5 \mathrm{~mm}$ chambers flushed with Argon gas for 4 different neutron source placements. Each run corresponds to a neutron flux as indicated in Table 1. 

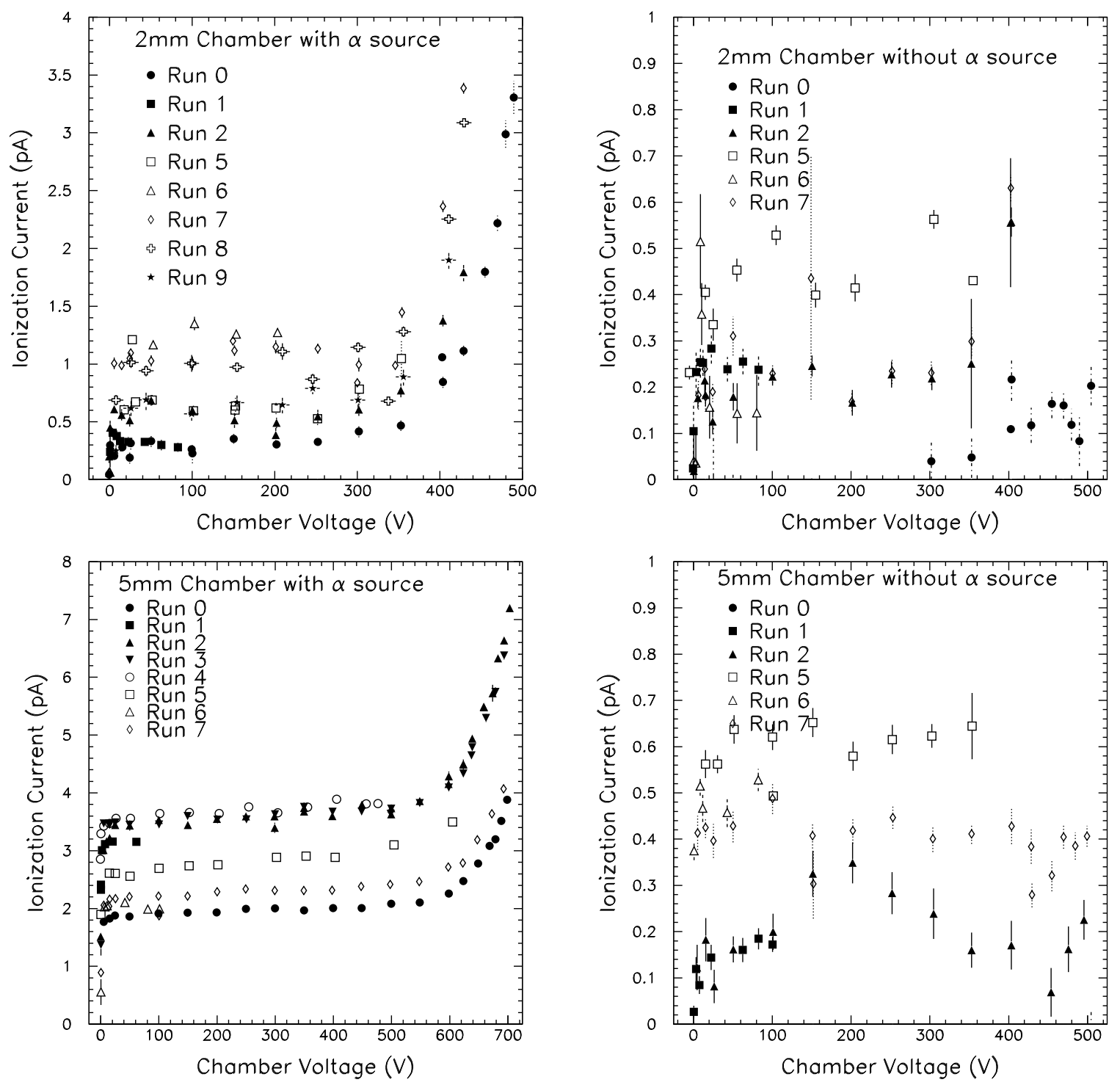

Fig. 4. Plateau curves for $2 \mathrm{~mm}$ and $5 \mathrm{~mm}$ chambers flushed with Helium gas for 9 different neutron source placements. Each run corresponds to a neutron flux as indicated in Table 1. 


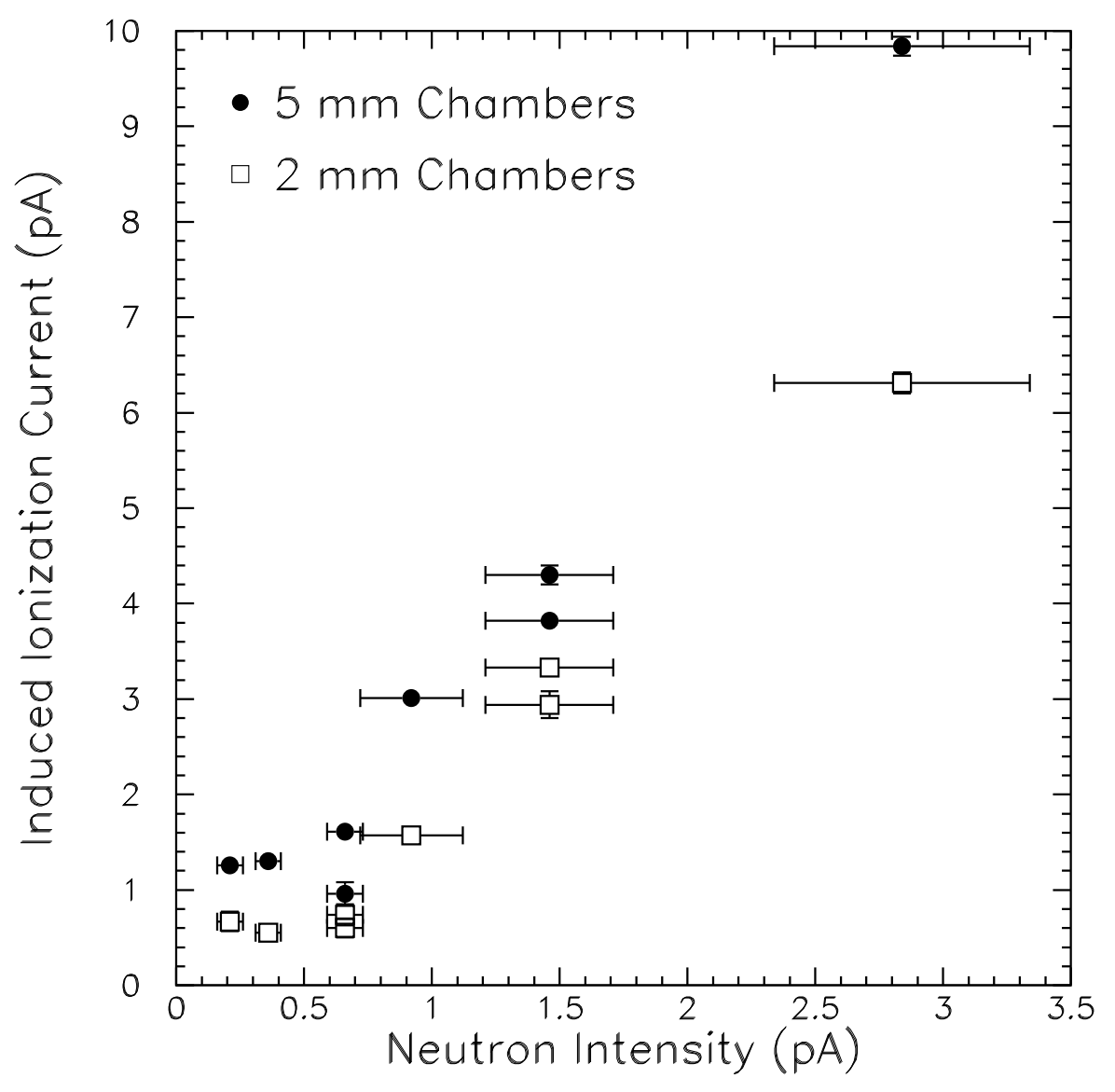

Fig. 5. Graph of the ionization current in Argon gas induced by the PuBe source as a function of neutron intensity. The neutron intensity is in units of 'pA', where $1 \mathrm{pA}=6.2 \times 10^{6}$ neutrons $/ \mathrm{sec}$. 


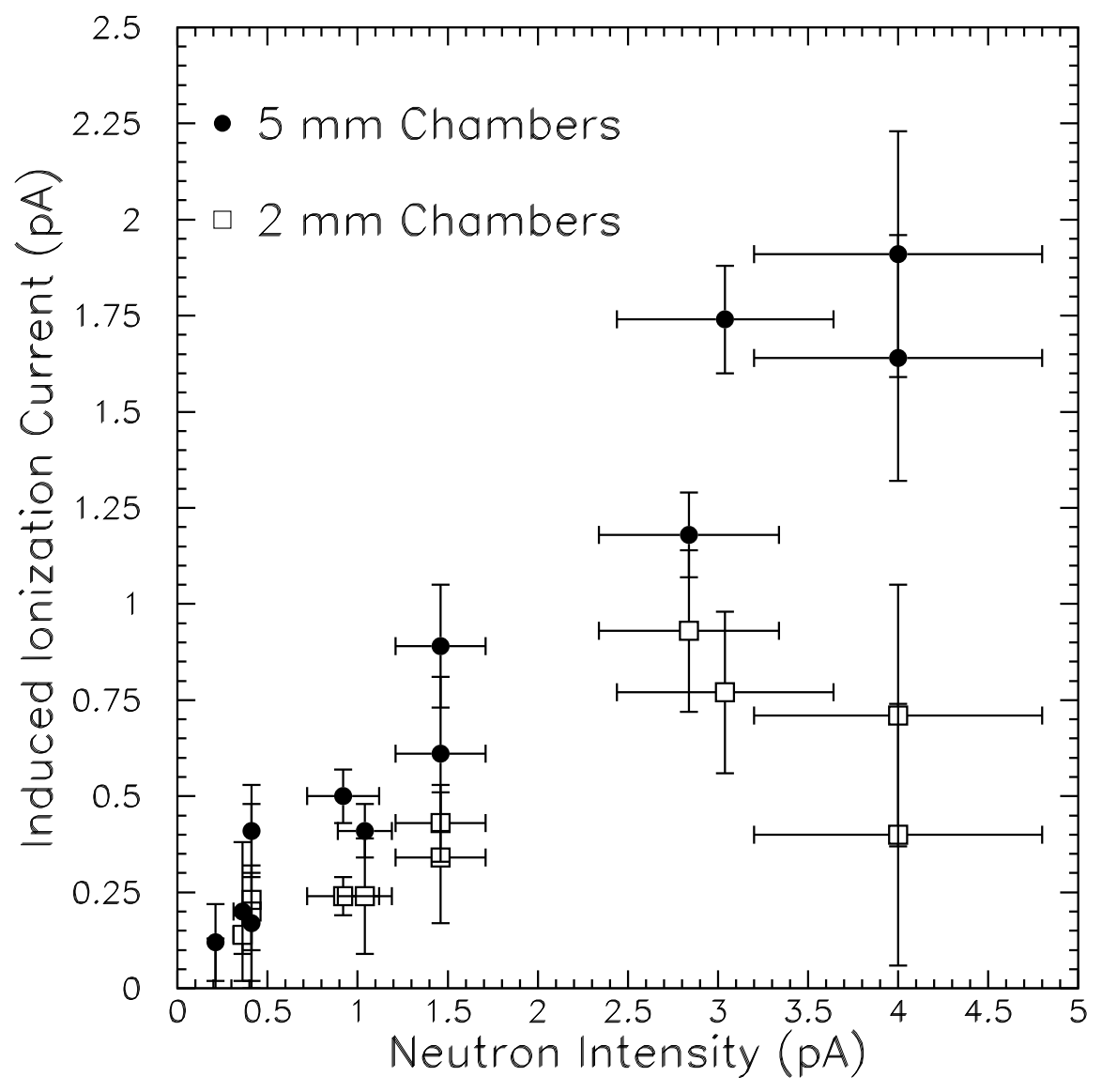

Fig. 6. Graph of the ionization current in Helium gas induced by the PuBe source as a function of neutron intensity. The neutron intensity is in units of 'pA', where $1 \mathrm{pA}=6.2 \times 10^{6}$ neutrons / sec. 